\title{
First-Principles Calculations of the Structural, Electronic and Magnetic Properties of Mn-Doped InSb by Using mBJ Approximation for Spintronic Application
}

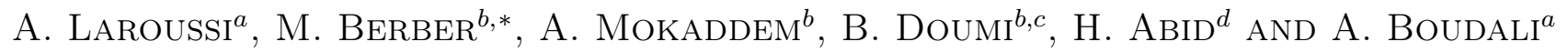 \\ ${ }^{a}$ Laboratory of Physico-Chemical Studies, University of Saida, 20000 Saida, Algeria \\ ${ }^{b}$ Laboratoire d'Instrumentation et Matériaux Avancés, Centre Universitaire Nour Bachir El-Bayadh, \\ BP 900 route Aflou, 32000, Algeria \\ ${ }^{c}$ Physics Department, Faculty of Science, University Dr. Tahar Moulay of Saida, Algeria \\ ${ }^{d}$ Applied Materials Laboratory, Research Center, Sidi Bel Abbès Djillali Liabes University, 22000, Algeria
}

(Received August 9, 2018; revised version January 20, 2019; in final form February 4, 2019)

In this work, we have used the first-principle calculations approach based on spin-polarized density functional theory, and predicted the half-metallic behavior of $\operatorname{In}_{1-x} \mathrm{Mn}_{x} \mathrm{Sb}$ alloys $(x=0.25,0.50,0.75)$. The structural, electronic and magnetic properties of these ternary cubic crystals have been investigated. The electronic exchangecorrelation energy has been characterized by the generalized gradient approximation with the WC functional improved by the Tran-Blaha modified Becke-Johnson exchange potential approximation. The calculated formation energy indicates that $\mathrm{In}_{1-x} \mathrm{Mn}_{x} \mathrm{Sb}$ compounds (at all compositions) are thermodynamically stable, and can be synthesized. Our calculated structural parameters are in good accordance with the available theoretical and experimental data. The ternary alloys $\operatorname{In}_{1-x} \mathrm{Mn}_{x} \mathrm{Sb}$ are half-metallic ferromagnets with a spin polarization at the Fermi level of $100 \%$. Our investigation shows an antiferromagnetic interaction between Mn-Sb and Mn-In, the total magnetic moment is equal to $4.00 \mu_{\mathrm{B}}$ for all studied structures. These materials are half-metallic ferromagnets, and thus may be potential candidates for spintronics applications.

DOI: 10.12693/APhysPolA.135.451

PACS/topics: GGA-WC, FP-LAPW, TB-mBJ, half metallic ferromagnetic

\section{Introduction}

In September 1925, Goudsmit and Uhlenbeck discovered the spin [1]. This discovery was a radical change for semiconductors, but some years later the magnetic structure for electrons led to spintronics. Spintronics or magnetoelectronics is important for applications such as to store information, nuclear magnetic resonance (NMR), electron spin resonance spectroscopy, and magnetic resonance imaging (MRI) in medicine. The real importance of spintronics was the giant magnetoresistance (GMR), discovered by Barnaś and Fert in ferromagnetic thin films [2] for drive head technology which are used in modern hard disks. The dilute magnetic semiconductors (DMSs) are semiconductors which can be doped with transition metals or rare earth elements, leading to a variety of cooperative effects. In the early 1970 s and late 1980s a new diluted magnetic III-V semiconductor $\operatorname{In}_{1-x} \mathrm{Mn}_{x}$ As (with a low concentration) has been produced by molecular-beam epitaxy (MBE) [3]. Recently, many researchers are working on diluted magnetic III-V and II-VI semiconductors for spintronics applications. Berber et al. have predicted the half-metallic ferromagnetism for $\mathrm{Sr}_{1-x} \mathrm{~V}_{x} \mathrm{O}$ and found that vanadium $(\mathrm{V})$ doped strontium oxide $(\mathrm{SrO})$ is a

*corresponding author; e-mail: berber.mohamed16@gmail.com half-metallic ferromagnet for a concentration $x=0.25$ and 0.50 with a half-metallic ferromagnetic gap $\left(G_{\mathrm{HMF}}\right)$ of $2.634,2.359 \mathrm{eV}$ and a half-metallic gap $\left(G_{\mathrm{HM}}\right)$ of 1.0 , $0.1 \mathrm{eV}$ respectively $[4,5]$. Berriah et al. studied halfmetallicity of $\mathrm{Cr}$ doped BaTe and predicted that for all concentrations of $\mathrm{Cr}$ the structure $\mathrm{Ba}_{1-x} \mathrm{Cr}_{x} \mathrm{Te}$ is a halfmetallic ferromagnet with a spin polarization of $100 \%[6]$. The considerable $s p-d$ exchange interaction between the electrons of the band and $\mathrm{Mn}^{2+}$, the DMs II-VI and III-V doped with manganese $(\mathrm{Mn})$ attracts many researchers. The prediction that was made [7] of Mn-doped AlSb $\left(\mathrm{Al}_{1-x} \mathrm{Mn}_{x} \mathrm{Sb}\right)$ is a half-metal ferromagnet for all concentrations (except for MnSb). The magnetic, magnetotransport and magneto-optical properties were studied by Ohno for Mn-doped GaAs and Mn-doped InAs [8].

In this context we have chosen to study the electronic and magnetic properties of the binary InSb doped with manganese $(\mathrm{Mn})$. In this study, we have predicted the structural, electronic and magnetic properties of $\operatorname{In}_{1-x} \mathrm{Mn}_{x} \mathrm{Sb}$ alloys with the concentrations $(x)$ $(x=0.25,0.50$ and 0.75$)$. For this prediction, we have utilized the full potential linearized augmented plane wave (FP-LAPW) method within density functional theory (DFT) [9]. For theoretical calculations of the structural constants we used the generalized gradient approximation GGA with the WC functional [10], but for the electronic and magnetic properties we used the TranBlaha modified Becke-Johnson exchange potential approximation (TB-mBJ) [11]. 
This paper is composed as follows: in Sect. 2, we describe the method of the calculation. In Sect. 3, we present the details of the collected results and discuss the structural, electronic, and magnetic properties of the $\mathrm{In}_{1-x} \mathrm{Mn}_{x} \mathrm{Sb}$ alloys. The main conclusions of our present work are summarized in Sect. 4.

\section{Computational detail}

In this paper, we have used the full-potential linearized augmented plane wave (FP-LAPW) method within the framework of the density functional theory (DFT) [9] as implemented in the WIEN2k code [12]. For computing the structural parameters, we have used the generalized gradient approximation (GGA) with the WC functional [10]. The electronic and magnetic properties of $\mathrm{In}_{1-x} \mathrm{Mn}_{x} \mathrm{Sb}$ are calculated using (GGA-WC), but with the new favored and improved method designed, known as the TB-mBJ potential approach [11] which enables us to perfectly represent the electronic structure of solids [13-15]. For the evaluation of the band gap, this theory has been proven by Tran [16] to be better than the GGA and LDA exchange correlation potential. In the present investigation, we have the muffin-tin radii (MT) for all atoms (In, Mn, and Sb) to be 2.50 atomic units (a.u.). The $K_{\max }=9\left(R_{\mathrm{MT}}\right)^{-1}\left(K_{\max }\right.$ is the plane wave cut-off and $R_{\mathrm{MT}}$ is the smallest of all atomic sphere radii). The Fourier expansion of the charge density was truncated at $G_{\max }=12(\mathrm{Ry})^{1 / 2}$, the $l$-expansion of the non-spherical potential and charge density was carried out up to $l_{\max }=10$. The cut-off energy is set to $-7 \mathrm{Ry}$ to separate the core from the valence states. The self-consistent calculations are evaluated to be converged when the total energy of the system is stable within $0.0001 \mathrm{Ry}$.

\section{Results and discussion}

\subsection{Stability and structural properties}

Indium antimonide (InSb) crystallizes in the zincblende structure (no. 216), in which the two types of atoms, In and $\mathrm{Sb}$, are located at $(0,0,0)$ and $(0.25$, $0.25,0.25)$ positions, respectively. We created a supercells with 8 atoms ( 4 atoms for In and 4 for Sb). We substituted one or three of these sites to create the structure of $\operatorname{In}_{0.75} \mathrm{Mn}_{0.25} \mathrm{Sb}$ and $\mathrm{In}_{0.25} \mathrm{Mn}_{0.75} \mathrm{Sb}$ with concentration $x=0.25$, and 0.75 , respectively. Then we created a supercells with 16 atoms ( 8 atoms for In and 8 for $\mathrm{Sb}$ ), and substituted In sites with Mn to create the structure $\mathrm{In}_{0.50} \mathrm{Mn}_{0.50} \mathrm{Sb}$. The $\mathrm{In}_{0.75} \mathrm{Mn}_{0.25} \mathrm{Sb}$ and $\mathrm{In}_{0.25} \mathrm{Mn}_{0.75} \mathrm{Sb}$ structures have a cubic structure with space group $P-43 \mathrm{~m}$ (no. 215), while $\operatorname{In}_{0.50} \mathrm{Mn}_{0.50} \mathrm{Sb}$ has a cubic structure in space group $F-43 m$ (no. 216) (zinc blende).

TABLE I

Lattice constant $a$, bulk modulus $B$, and its pressure derivative $B^{\prime}$ for $\operatorname{InSb}$ and $\operatorname{In}_{1-x} \mathrm{Mn}_{x} \mathrm{Sb}_{\text {with }}$ composition $x=0.25$, 0.5 and 0.75 .

\begin{tabular}{l|c|c|c|c}
\hline \hline Compound & $a[\AA]$ & $B$ & $B^{\prime}$ & Method \\
\hline $\mathrm{InSb}_{\mathrm{In}}$ & $6.5555,6.464^{a}, 6.36^{a}, 6.479^{b}, 6.629^{c}$ & $42.4612,37.368^{c}$ & $4.0525,4.452^{c}$ & GGA-WC \\
$\mathrm{In}_{0.50} \mathrm{Mn}_{0.50} \mathrm{Sb}$ & 6.4363 & 44.3600 & 6.0030 & GGA-WC \\
$\mathrm{In}_{0.25} \mathrm{Mn}_{0.75} \mathrm{Sb}$ & 6.3339 & 48.0342 & 4.2197 & GGA-WC \\
\hline
\end{tabular}

${ }^{a}$ Ref. [18], ${ }^{b}$ Ref. [19], ${ }^{c}$ Ref. [20].

After optimization of all structures of $\operatorname{In}_{1-x} \mathrm{Mn}_{x} \mathrm{Sb}$ with $x=0,0.25,0.50,0.75,1$, we calculated the total energies as a functional the unit cell volumes and fitted the results with the Birch-Murnaghan equation of state (EOS) [17] to evaluate the ground state properties such as the equilibrium lattice constant $a$, the bulk modulus $B_{0}$ and the bulk modulus pressure derivative $B^{\prime}$. The studied structural parameters of $\operatorname{In}_{1-x} \mathrm{Mn}_{x} \mathrm{Sb}$ for various concentrations are shown in Table I including the available theoretical and experimental data. Table I shows that our results are improved compared with the results of Verma et al. [20].

The variation of lattice constant $a$ as a function of the concentration $(x)$ for $\operatorname{In}_{1-x} \mathrm{Mn}_{x}$ Sb is illustrated in Fig. 1. This variation of the calculated lattice parameter versus concentration $x$ is fitted to a polynomial of second order

$$
a(x)=6.55358-0.43389 x-0.04011 x^{2} .
$$

The thermodynamic stability for the synthesis of the alloys is determined by the energy of formation [21, 22]. In order to obtain the phase stability of the $\operatorname{In}_{0.75} \mathrm{Mn}_{0.25} \mathrm{Sb}$, $\mathrm{In}_{0.50} \mathrm{Mn}_{0.50} \mathrm{Sb}, \quad \mathrm{In}_{0.25} \mathrm{Mn}_{0.75} \mathrm{Sb}$ structures, we computed the formation energies using the following formula $[23,24]$ :

$$
\begin{aligned}
& E_{f}= \\
& \quad E_{\mathrm{In}_{1-x} \mathrm{Mn}_{x} \mathrm{Sb}}^{\mathrm{total}}-\left[E_{\mathrm{Sb}}^{\mathrm{Bulk}}+x E_{\mathrm{Mn}}^{\mathrm{Bulk}}+(1-x) E_{\mathrm{In}}^{\mathrm{Bulk}}\right],
\end{aligned}
$$

where $E_{\operatorname{In}_{1-x} \mathrm{Mn}_{x} \mathrm{Sb}}^{\text {total }}$ is the total energy per atom of $\mathrm{In}_{1-x} \mathrm{Mn}_{x} \mathrm{Sb}, x$ is the concentration of $\mathrm{Mn}$ in the supercell. The $E_{\mathrm{Sb}}^{\mathrm{Bulk}}, E_{\mathrm{Mn}}^{\mathrm{Bulk}}$, and $E_{\mathrm{In}}^{\mathrm{Bulk}}$ are the total energies of bulk $\mathrm{Sb}, \mathrm{Mn}$, and In, respectively. Our calculated values of the formation energy for the ternary alloys are -2.35 , 


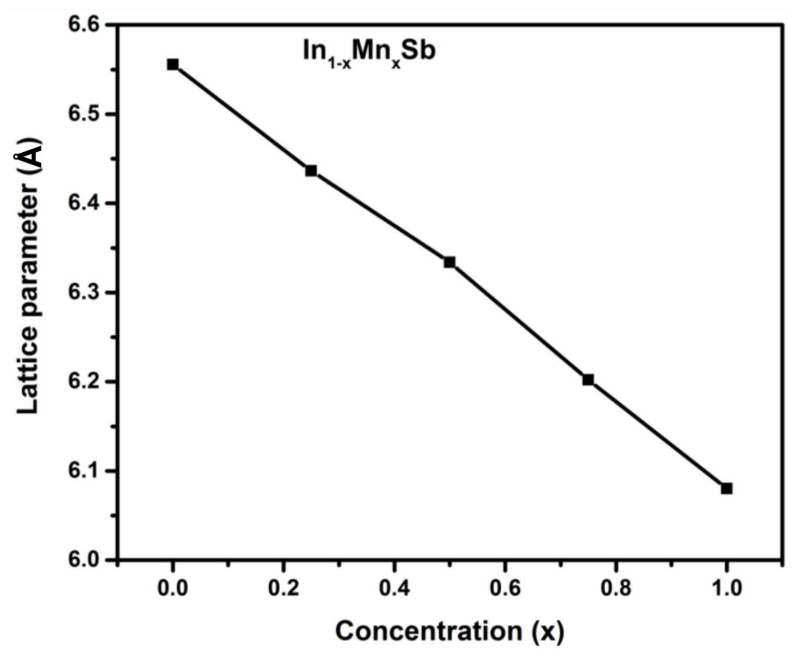

Fig. 1. Calculated lattice constant $a$ of $\operatorname{In}_{1-x} \mathrm{Mn}_{x} \mathrm{Sb}$ as a function of concentration $x$.

-2.04 , and $-3.18 \mathrm{eV}$ for $\operatorname{In}_{0.75} \mathrm{Mn}_{0.25} \mathrm{Sb}, \mathrm{In}_{0.50} \mathrm{Mn}_{0.50} \mathrm{Sb}$, and $\mathrm{In}_{0.25} \mathrm{Mn}_{0.75} \mathrm{Sb}$, respectively. Therefore, the negative values of formation energies signify that the ternary alloys $\operatorname{In}_{1-x} \mathrm{Mn}_{x} \mathrm{Sb}(x=0.25,0.50$ and 0.75$)$ are thermodynamically stable and can be synthesized.

\subsection{Electronic, magnetic properties and half-metallic character}

In this part, we present the electronic band structure, total and partial densities of states for $\operatorname{In}_{1-x} \mathrm{Mn}_{x} \mathrm{Sb}$ with different concentration $x(x=0,0.25,0.50,0.75,1)$ using the GGA-WC [10] of TB-mBJ [11] approaches. The band structures of the alloys of $\mathrm{In}_{1-x} \mathrm{Mn}_{x} \mathrm{Sb}$ are shown in Figs. 2-6 with the concentrations 0, 0.25, 0.50, 0.75, and 1 , respectively. Since $\mathrm{InSb}$ is non magnetic, the two spin channels of InSb have the same semiconducting band structures with a direct band gap $\left(E_{g}^{\Gamma-\Gamma}\right)$ located at the $\Gamma$ point. The spin polarized band structures of

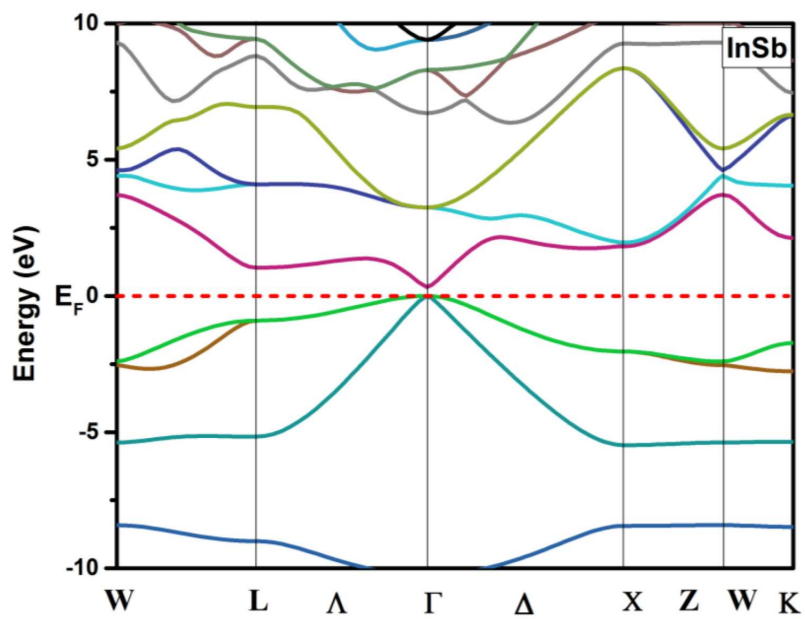

Fig. 2. Spin-polarized band structure obtained with TB-mBJ for InSb: the Fermi level is set to zero (horizontal dotted red line).
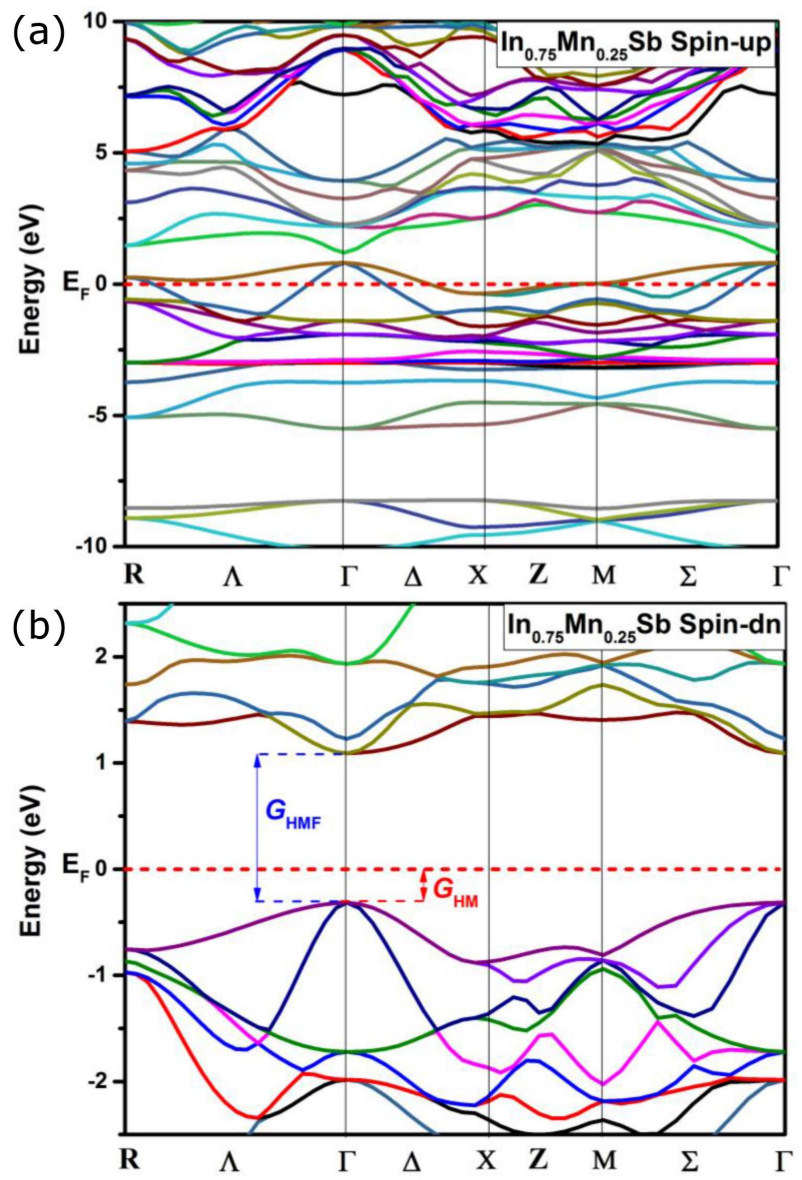

Fig. 3. Spin-polarized band structure obtained with TB-mBJ for $\operatorname{In}_{0.75} \mathrm{Mn}_{0.25} \mathrm{Sb}$ : (a) majority spin (up), and (b) minority spin (dn). The Fermi level is set to zero (horizontal dotted red line).

$\mathrm{In}_{1-x} \mathrm{Mn}_{x} \mathrm{Sb}$ (at concentrations of $x=0.25,0.50,0.75$, and 1) are presented in Figs. 3-6. We remark that the majority-spin bands are metallic whereas a gap is located at the Fermi level for the minority-spin bands. For the minority-spin, we notice two gaps, the half-metallic ferromagnetic gap $\left(G_{H M F}\right)$ and the half-metallic gap $\left(G_{H M}\right)$. The $G_{\mathrm{HMF}}$ is the energy difference between the valenceband maximum (VBM) and conduction-band minimum (CBM), while $G_{\mathrm{HM}}$ is the (smaller) energy between the Fermi level and the maximum value of the valence band energy $[25,26]$.

Table II illustrates the computed direct gap $\left(E_{g}^{\Gamma-\Gamma}\right)$ of $\mathrm{InSb}$, and the HMF $\left(G_{H M F}\right)$ and HM $\left(G_{\mathrm{HM}}\right)$ gaps of $\mathrm{In}_{1-x} \mathrm{Mn}_{x} \mathrm{Sb}$ at all compositions $(x)$, using the TBmBJ exchange potential, together with other experimental and theoretical data [20]. Figures 3 and 4 show that $\mathrm{In}_{0.75} \mathrm{Mn}_{0.25} \mathrm{Sb}$ and $\mathrm{In}_{0.50} \mathrm{Mn}_{0.50} \mathrm{Sb}$ have a direct halfmetallic ferromagnetic gap $G_{\mathrm{HMF}}$ of 1.4085 and $1.3192 \mathrm{eV}$ at the $\Gamma$ high-symmetry point and exhibit a half-metallic gap $G_{\mathrm{HM}}$ of 0.3161 and $0.4769 \mathrm{eV}$, respectively. However, Fig. 5 illustrates that $\mathrm{In}_{0.25} \mathrm{Mn}_{0.75} \mathrm{Sb}$ shows an indirect half-metallic ferromagnetic gap between $R$ and $\Gamma$ highsymmetry points of $1.4503 \mathrm{eV}$ and a half-metallic gap of 

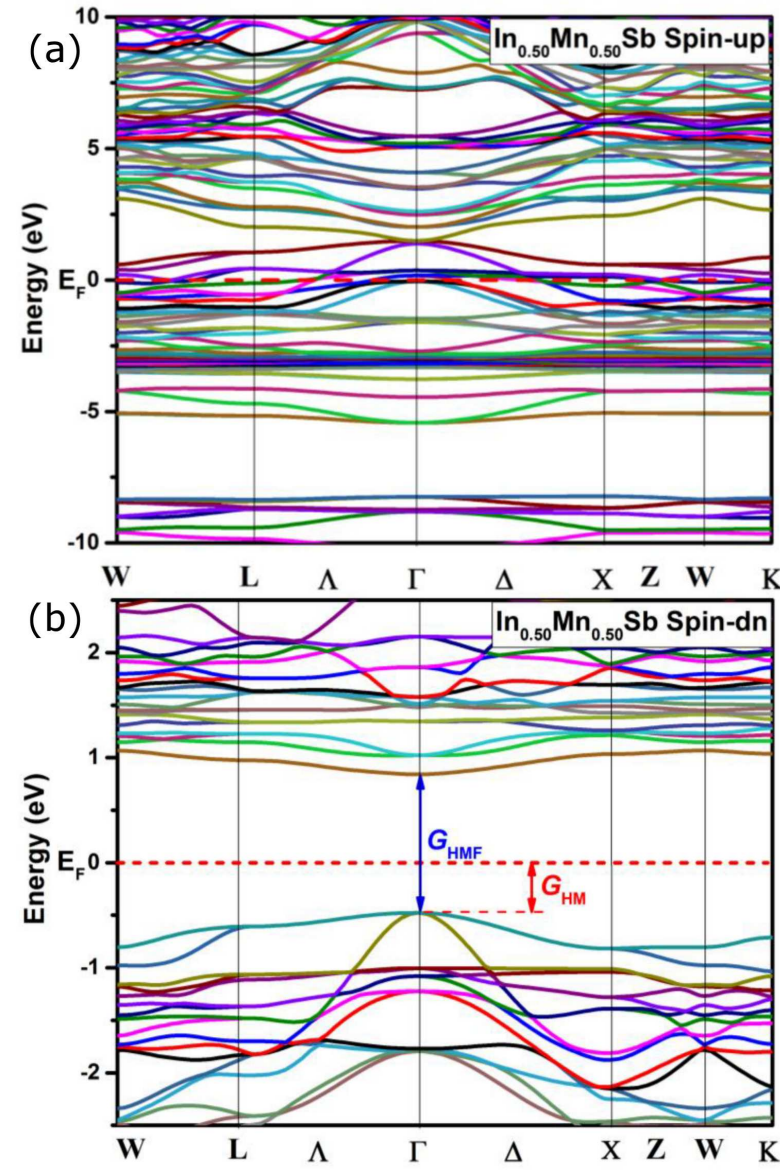

Fig. 4. As in Fig. 3, but for $\operatorname{In}_{0.50} \mathrm{Mn}_{0.50} \mathrm{Sb}$.

$0.4140 \mathrm{eV}$. This makes them promising materials for spintronic applications. In summary, Table II shows that our results have improved the results of Verma et al. [20].

The densities of states (DOS) near the Fermi level $E_{F}$ describe the metallic or semiconductor behaviour of these structures, based on the ferromagnetic disposition; we have also calculated the spin-polarized total DOS (TDOS) and partial densities of states (PDOS) for $\mathrm{In}_{1-x} \mathrm{Mn}_{x} \mathrm{Sb}$ for different compositions $(x)$. The spinpolarized (TDOS) and (PDOS) of InSb, $\operatorname{In}_{0.75} \mathrm{Mn}_{0.25} \mathrm{Sb}$, $\mathrm{In}_{0.50} \mathrm{Mn}_{0.50} \mathrm{Sb}$, and $\mathrm{In}_{0.25} \mathrm{Mn}_{0.75} \mathrm{Sb}$ are demonstrated in Figs. 6-9, respectively. For InSb, the spin-up and spindown states are the same with a semiconductor character, and this non-magnetic type of this compound is exposed in Fig. 6. On the other hand, Figs. 7-9 exhibit the TDOS of $\operatorname{In}_{1-x} \mathrm{Mn}_{x}$ Sb for all concentrations with different band states for spin-up and spin-down, caused by the different $p-d$ hybridization between the $\mathrm{Sb} p$ and Mn $3 d$ states around the Fermi level for the two directions. The minority-spin states (for all compositions) show a gap around the Fermi level, and thus $\mathrm{In}_{0.75} \mathrm{Mn}_{0.25} \mathrm{Sb}, \mathrm{In}_{0.50} \mathrm{Mn}_{0.50} \mathrm{Sb}$, and $\mathrm{In}_{0.25} \mathrm{Mn}_{0.75} \mathrm{Sb}$ alloys are half-metallic ferromagnets with a spin polarization of $100 \%$ according to the following expression [27]:
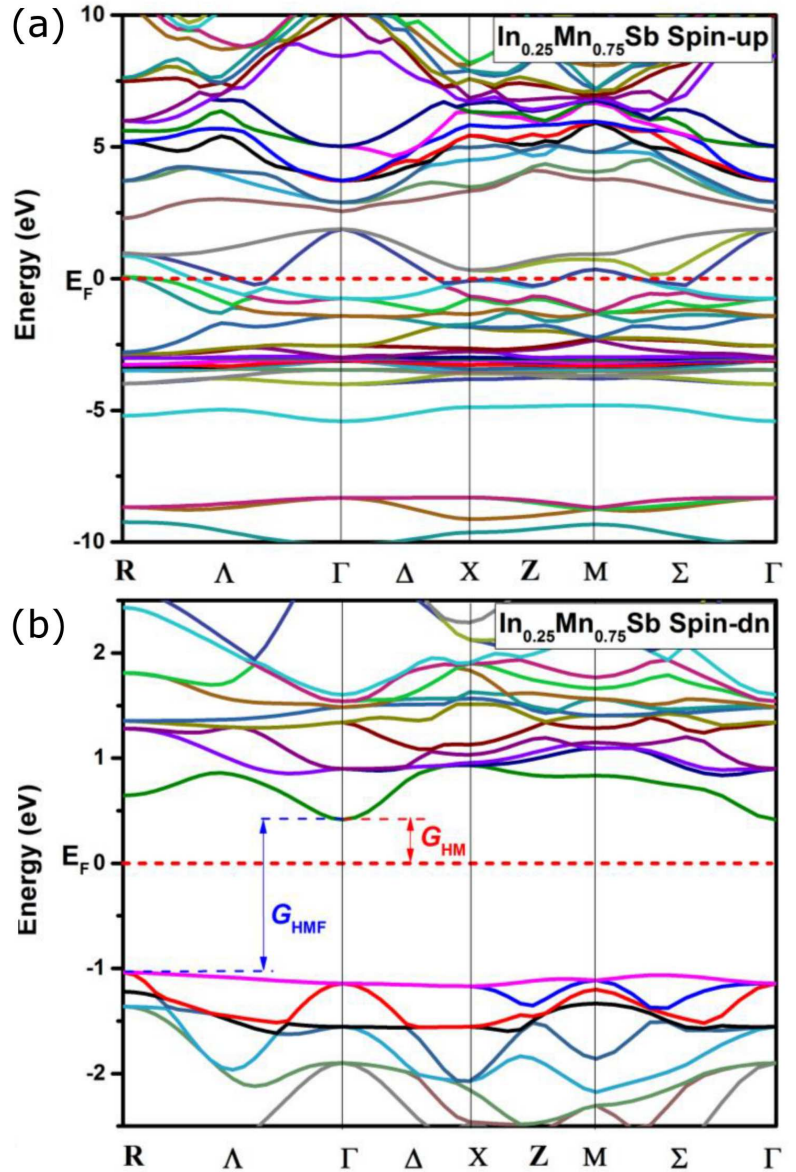

Fig. 5. As in Fig. 3, but for $\operatorname{In}_{0.25} \mathrm{Mn}_{0.75} \mathrm{Sb}$.

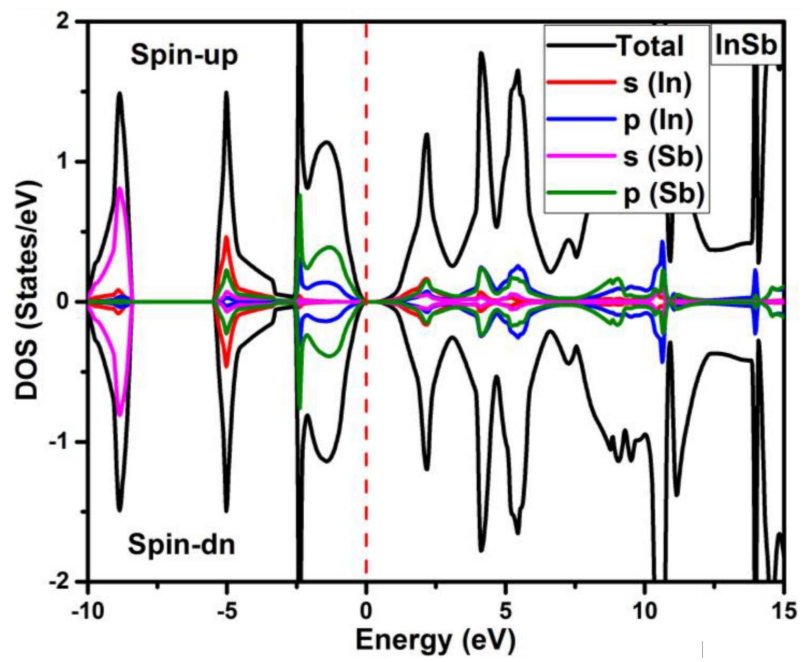

Fig. 6. Spin-polarized total densities of states of InSb. The Fermi level is set to zero (vertical dotted red line).

$$
P=\frac{N \uparrow\left(E_{\mathrm{F}}\right)-N \downarrow\left(E_{\mathrm{F}}\right)}{N \uparrow\left(E_{\mathrm{F}}\right)+N \downarrow\left(E_{\mathrm{F}}\right)} 100 \% .
$$

$P$ is the spin polarization, where $N \uparrow\left(E_{\mathrm{F}}\right)$ and $N \downarrow\left(E_{\mathrm{F}}\right)$ are the densities of states of the majority and minority spin around the Fermi level, respectively. We observe 
TABLE II

Calculated half-metallic ferromagnetic gap $\left(G_{\mathrm{HMF}}\right)$ and half-metallic gap $\left(G_{\mathrm{HM}}\right)$ of minority-spin band for $\operatorname{In}_{1-x} \mathrm{Mn}_{x} \mathrm{Sb}$ with composition $x=0.25,0.5$, and 0.75 and direct band gap $E_{g}^{\Gamma-\Gamma}$ for $\operatorname{InSb}$.

\begin{tabular}{l|c|c|c|c|c}
\hline \hline Compound & $G_{\text {HMF }}[\mathrm{eV}]$ & $G_{\text {HM }}[\mathrm{eV}]$ & $E_{g}^{\Gamma-\Gamma}$ & Method & Behavior \\
\hline $\mathrm{InSb}$ & - & - & $0.3216,0.24^{b}$ & TB-mBJ & \\
$\mathrm{In}_{0.75} \mathrm{Mn}_{0.25} \mathrm{Sb}$ & $1.4085,0.328^{c}$ & $0.3161,0.045^{c}$ & & TB-mBJ & HMF \\
$\mathrm{In}_{0.50} \mathrm{Mn}_{0.50} \mathrm{Sb}$ & $1.3192,0.885^{c}$ & $0.4769,0.395^{c}$ & & TB-mBJ & HMF \\
$\mathrm{In}_{0.25} \mathrm{Mn}_{0.75} \mathrm{Sb}$ & $1.4503,0.961^{c}$ & $0.4140,0.250^{c}$ & & TB-mBJ & HMF \\
\hline
\end{tabular}

\footnotetext{
${ }^{b}$ Ref. [19], ${ }^{c}$ Ref. [20].
}
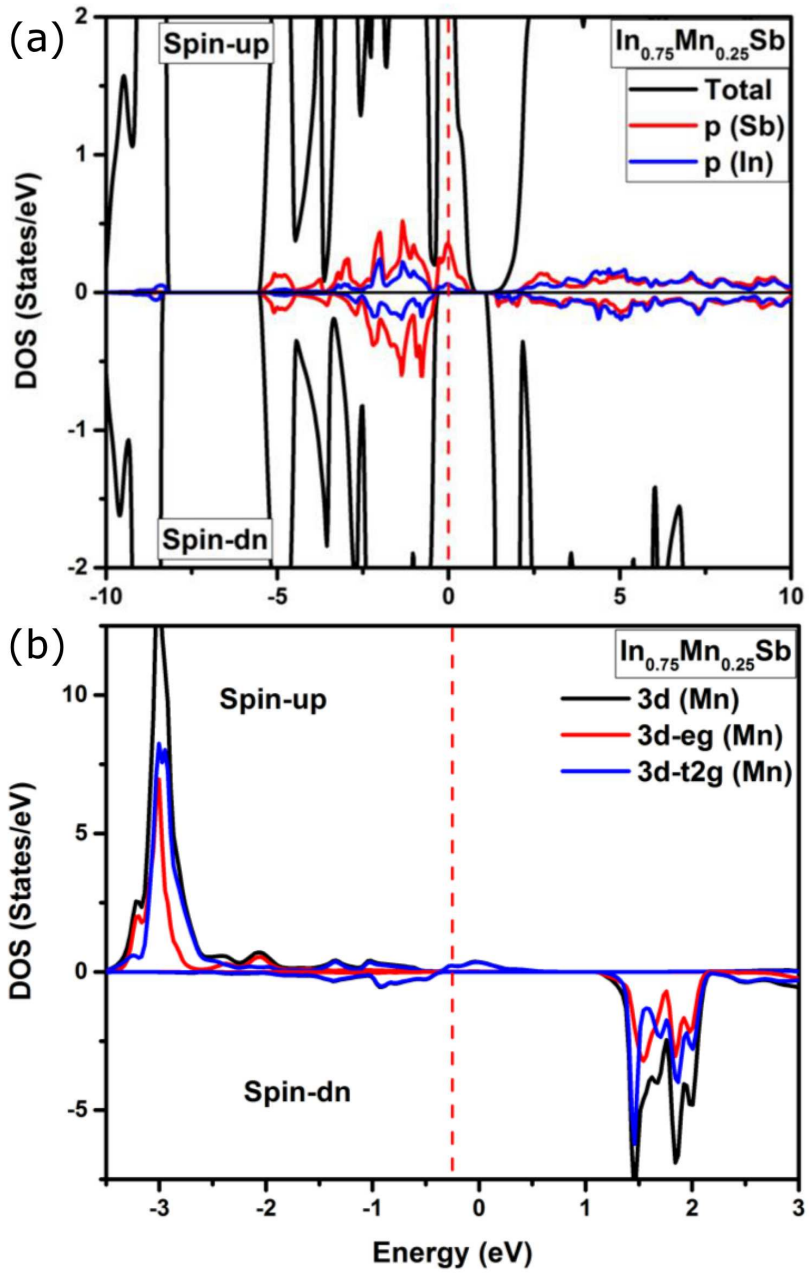

Fig. 7. Spin-polarized (a) total and (b) partial densities of states of $\operatorname{In}_{0.75} \mathrm{Mn}_{0.25} \mathrm{Sb}$. The Fermi level is set to zero (vertical dotted red line).

that the top section of the valence bands is primarily composed of Mn 3d partially occupied states, while the lowest part of the conduction bands is controlled by the Mn $3 d$ unoccupied states, which are higher than the Fermi level $E_{F}$.

Finally, Table III exhibits the total and local magnetic moments of $\operatorname{In}_{0.75} \mathrm{Mn}_{0.25} \mathrm{Sb}, \mathrm{In}_{0.50} \mathrm{Mn}_{0.50} \mathrm{Sb}$, and $\mathrm{In}_{0.25} \mathrm{Mn}_{0.75} \mathrm{Sb}$. We calculated the total and local magnetic moments of the relevant $\mathrm{Mn}$, In, and Sb atoms,
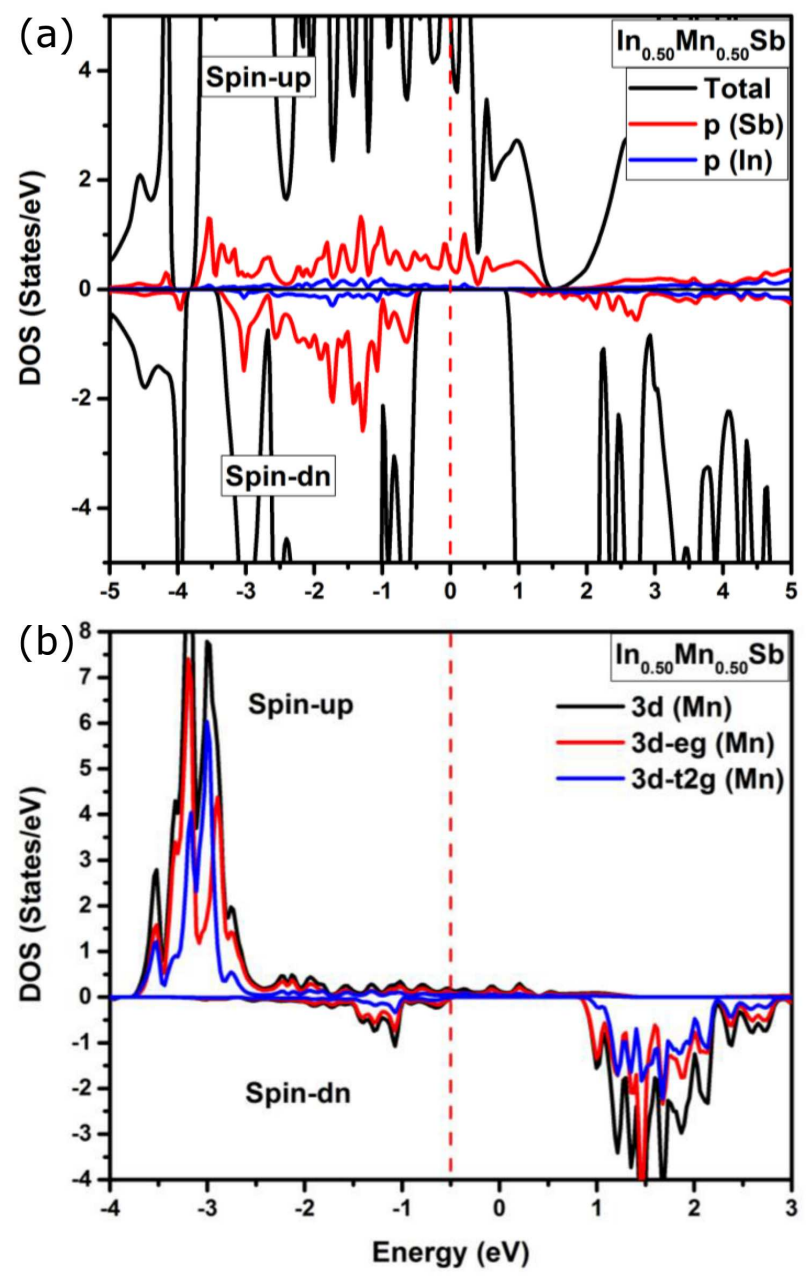

Fig. 8. As in Fig. 7, but for $\operatorname{In}_{0.50} \mathrm{Mn}_{0.50} \mathrm{Sb}$.

and at the interstitial region in the $\mathrm{In}_{1-x} \mathrm{Mn}_{x} \mathrm{Sb}$ structures in order to get the details of the $p-d$ exchange system on the magnetic properties. The results show that the total magnetic moment for all compounds was $4 \mu_{B}$, dominated by the local magnetic moment of the Mn atom. The Mn 3d majority-spin states, which are partially filled with five electrons, cause a total magnetic moment of $4 \mu_{B}$. On the other hand, the simply predicted magnetic moment of the $\mathrm{Mn}$ atom is reduced to below $4 \mu_{B}$ and minor local magnetic moments are 

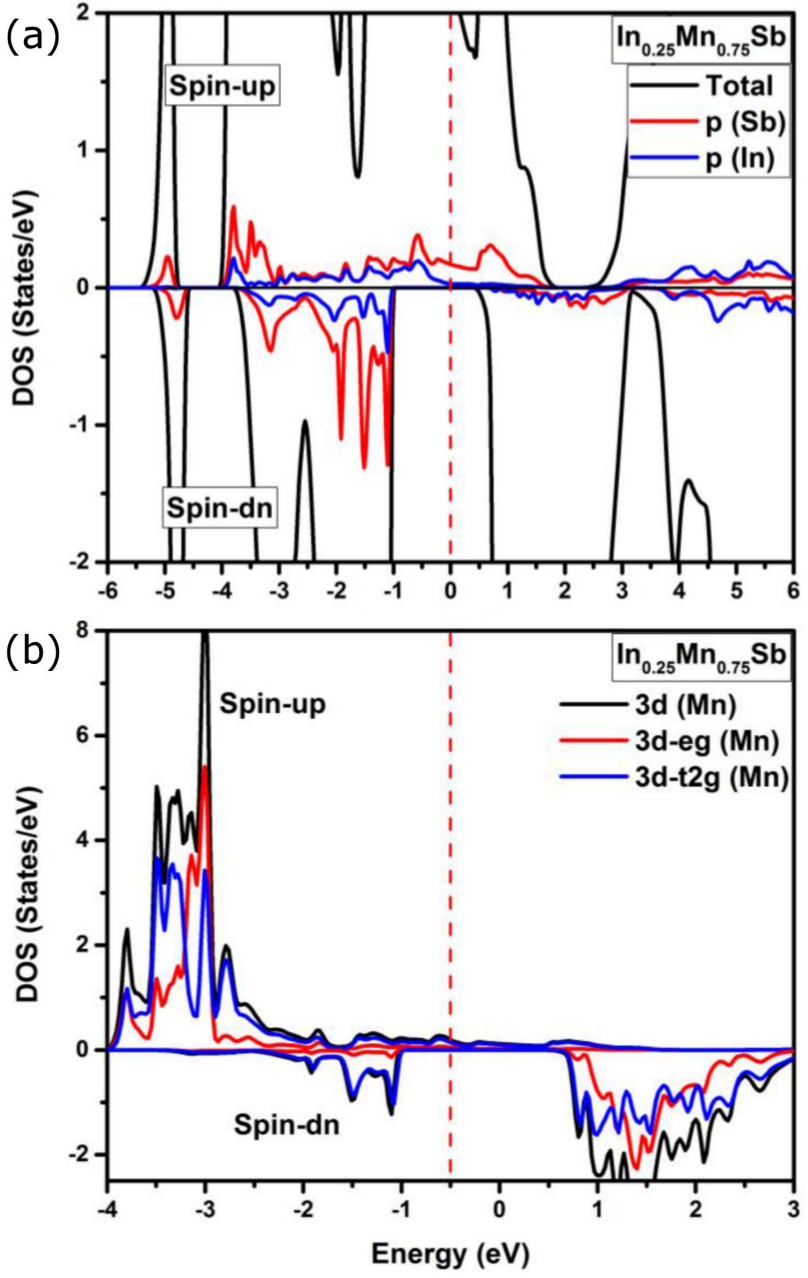

Fig. 9. As in Fig. 7, but for $\operatorname{In}_{0.25} \mathrm{Mn}_{0.75} \mathrm{Sb}$. induced at $\mathrm{In}, \mathrm{Sb}$, and the interstitial regions due to the $p-d$ exchange interaction between the $\mathrm{Sb} p$ and Mn $3 d$ levels. Table III shows that our results have improved the results of Verma et al. [20]. It also indicates that for all compositions of $\operatorname{In}_{1-x} \mathrm{Mn}_{x} \mathrm{Sb}$, not only the magnetic moments of the $\mathrm{Mn}$ and $\mathrm{Sb}$ but also of $\mathrm{Mn}$ and In have opposite signs, indicating an antiferromagnetic interaction between $\mathrm{Mn}$ and $\mathrm{Sb}$ and between $\mathrm{Mn}$ and In.

\section{Conclusion}

In this study, we have used the first-principle calculations of the structural, electronic, and magnetic properties of the $\mathrm{In}_{1-x} \mathrm{Mn}_{x} \mathrm{Sb}$ alloys $(x=0.25,0.50$ and 0.75$)$. We have exploited the GGA-WC exchange correlation energy and improved it with the TB-mBJ approximation. The calculated formation energy indicates that the $\operatorname{In}_{1-x} \mathrm{Mn}_{x} \mathrm{Sb}$ compounds (for all section compositions) are thermodynamically stable, and can be synthesized. The computed lattice constant and energy band gap for InSb are in good agreement with the available experimental and theoretical data. The examination of the band structure and the density of states determine the half-metallic behaviour of $\mathrm{In}_{1-x} \mathrm{Mn}_{x} \mathrm{Sb}$ for all concentrations. The decomposition of the calculated total magnetic moment shows an antiferromagnetic interaction between $\mathrm{Mn}$ and $\mathrm{Sb}$ and between $\mathrm{Mn}$ and $\mathrm{In}$. Therefore, the $\mathrm{In}_{1-x} \mathrm{Mn}_{x} \mathrm{Sb}$ compounds are suitable candidates for possible spintronics applications, and thus we hope that our results can serve as reference for theoretical and experimental studies in future.

TABLE III

Calculated total and local magnetic moments (in the Bohr magneton $\mu_{\mathrm{B}}$ ) within the muffin-tin spheres and in the interstitial sites for $\operatorname{In}_{1-x} \mathrm{Mn}_{x}$ Sb with composition $x=0.25,0.5$, and 0.75 .

\begin{tabular}{c|c|c|c|c|c}
\hline \hline Compound & Total $\left[\mu_{B}\right]$ & $\operatorname{In}\left[\mu_{B}\right]$ & $\operatorname{Mn}\left[\mu_{B}\right]$ & Sb $\left[\mu_{B}\right]$ & Interstitial $\left[\mu_{B}\right]$ \\
\hline $\operatorname{In}_{0.75} \mathrm{Mn}_{0.25} \mathrm{Sb}$ & $4,4.00975^{c}$ & $0.01781,0.0049^{c}$ & $3.86920,3.8074^{c}$ & $-0.05002,-0.0087^{c}$ & $0.26823,0.7994^{c}$ \\
$\mathrm{In}_{0.50} \mathrm{Mn}_{0.50} \mathrm{Sb}$ & $4,4.001475^{c}$ & $0.02340,0.0195^{c}$ & $4.01154,3.7579^{c}$ & $-0.35069,-0.0106^{c}$ & $0.76202,1.6796^{c}$ \\
$\mathrm{In}_{0.25} \mathrm{Mn}_{0.75} \mathrm{Sb}$ & $4,4.00185^{c}$ & $0.06886,0.0405^{c}$ & $3.94111,3.7131^{c}$ & $-0.10514,-0.0768^{c}$ & $0.71483,2.7009^{c}$ \\
\hline
\end{tabular}

${ }^{c}$ Ref. [20].

\section{References}

[1] G.E. Uhlenbeck, S. Goudsmit, Nature 117, 264 (1926).

[2] J. Barnaś, A. Fert, Phys. Rev. Lett. 80, 1058 (1998).

[3] H. Munekata, H. Ohno, S. von Molnar, A. Segmüller, L.L. Chang, L. Esaki, Phys. Rev. Lett. 63, 1849 (1989).

[4] M. Berber, B. Doumi, A. Mokaddem, Y. Mogulkoc, A. Sayede, A. Tadjer, J. Electron. Mater. 47, 449 (2018).
[5] M. Berber, B. Doumi, A. Mokaddem, Y. Mogulkoc, A. Sayede, A. Tadjer, J. Computat. Electron. 16, 542 (2017).

[6] K. Berriah, B. Doumi, A. Mokaddem, M. Elkeurti, A. Sayede, A. Tadjer, J.P. Araújo, J. Computat. Electron. 17, 909 (2018).

[7] G. Rahman, S. Cho, S.C. Hong, Phys. Status Solidi $B$ 244, 4435 (2007).

[8] H. Ohno, J. Magn. Magn. Mater. 200, 110 (1999).

[9] W. Kohn, L.J. Sham, Phys. Rev. 140, A1133 (1965). 
[10] Z. Wu, R.E. Cohen, Phys. Rev. B 73, 235116 (2006).

[11] F. Tran, P. Blaha, Phys. Rev. Lett. 102, 226401 (2009).

[12] P. Blaha, K. Schwarz, G.K.H. Madsen, Comput. Phys. Commun. 147, 71 (2002).

[13] O. Yassin, Optik Int. J. Light Electron. Opt. 127, 1817 (2016).

[14] N.N. Anua, R. Ahmed, A. Shaari, M.A. Saeed, B.U. Haq, S. Goumri-Said, Semicond. Sci. Technol. 28, 105015 (2013).

[15] B.U. Haq, R. Ahmed, F.E.H. Hassan, R. Khenata M.K. Kasmin, S. Goumri-Said, Sol. Energy. 100, 1 (2014).

[16] F. Tran, P. Blaha, K. Schwarz, J. Phys. Condens. Matter. 19, 196208 (2007).

[17] F. Birch, Phys. Rev. 71, 809 (1947).

[18] S. Massidda, A. Continenza, A.J. Freeman, Phys. Rev. B 41, 12079 (1990).

[19] W.A. Harrison, Electronic Structure and the Properties of Solids, Freeman, San Francisco 1980, p. 157.
[20] U.P. Verma, N. Devi, S. Sharma, P. Jensen, Eur. Phys. J. B 81, 381 (2011).

[21] P. Reunchan, N. Umezawa, S. Ouyang, J. Ye, Phys. Chem. Chem. Phys. 14, 1876 (2012).

[22] Y. Liu, W. Zhou, P. Wu, J. Appl. Phys. 121, 075102 (2017).

[23] J. Bai, J.-M. Raulot, Y. Zhang, C. Esling, X. Zhao, L. Zuo, Appl. Phys. Lett. 98, 164103 (2011).

[24] B. Doumi, A. Mokaddem, M. Ishak-Boushaki, D. Bensaid, Sci. Semicond. Process. 32, 166 (2015).

[25] G.Y. Gao, K.L. Yao, E. Şaşıŏlu, L.M. Sandratskii, Z.L. Liu, J.L. Jiang, Phys. Rev. B 75, 174442 (2007).

[26] B. Doumi, A. Mokaddem, F. Dahmane, A. Sayede, A. Tadjer, $R S C$ Adv. 5, 92328 (2015).

[27] R.J. Soulen, Jr., J.M. Byers, M.S. Osofsky, B. Nadgorny, T. Ambrose, S.F. Cheng, P.R. Broussard, C.T. Tanaka, J. Nowak, J.S. Moodera, A. Barry, J.M.D. Coey, Science 282, 85 (1998). 